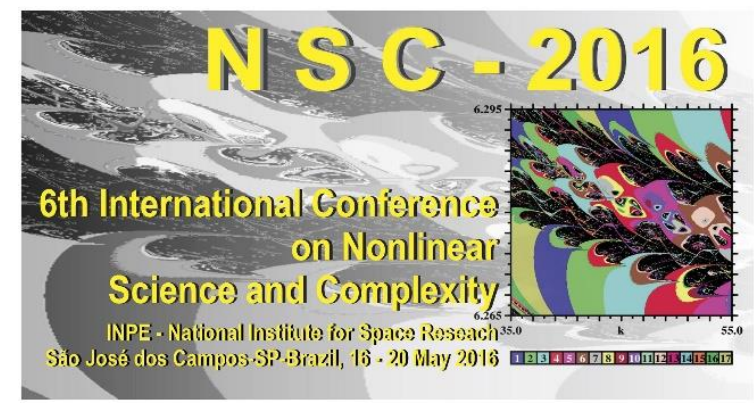

\author{
INPE - National Institute for Space Research \\ São José dos Campos - SP - Brazil - May 16-20, 2016
}

\title{
SUPERNOVAE AUTOMATIC CLASSIFICATION METHOD BY MODELING HUMAN ANALYSIS USING ARTIFICIAL NEURAL NETWORKS
}

\author{
Marcelo Módolo', Lamartine Nogueira Frutuoso Guimarães ${ }^{2}$, Reinaldo Roberto Rosa ${ }^{3}$ \\ 1 National Institute for Space Research, São José dos Campos, Brazil, marmodolo@hotmail.com \\ 2 Institute for Advanced Studies of the Department of Aerospace Science and Technology (IEAv/DCTA), São José dos \\ Campos, Brazil, guimarae@ieav.cta.br \\ 3 National Institute for Space Research, São José dos Campos, Brazil, reinaldo@lac.inpe.br
}

\begin{abstract}
The existing supernovae automatic classifiers do not models the human way of analyzing the spectrum. The automatic method proposed in this work takes into consideration the human way of classification using a Multilayer Perceptron neural network to classify the supernovae spectra. The experiments performed obtained significant results indicating the viability of using this method in places that require an automatic analysis or that have no specialist.
\end{abstract}

Keywords: Celestial mechanics and astronomy dynamics; modeling, numerical simulation and optimization; nonlinear systems and neural dynamics; supernovae automatic classification; supernovae spectrum analysis.

\section{INTRODUCTION}

The origin of a supernova is a star explosion. It results a great light emission that grows for about two weeks until reaching full brightness, then decreases gradually for about one year [1]. Research about supernovae are important because they help to understand the phenomenon of accelerated expansion of the universe. However, not all supernovae may be used in these studies. Only the type la supernovae are appropriate because it has high brightness and brightness dispersion is relatively small from its maximum brightness [2].

The supernovae classification is carried out since 1941 and, in addition to type la supernovae, classification schemes currently utilized, considers eight supernovae types more. Figure 1 shows the Giunt and Kin [1] classification scheme with the nine supernovae types: Ia, Ib, Ic, Ilb, IIL, IIP, IIP, IIn, Ilpec.

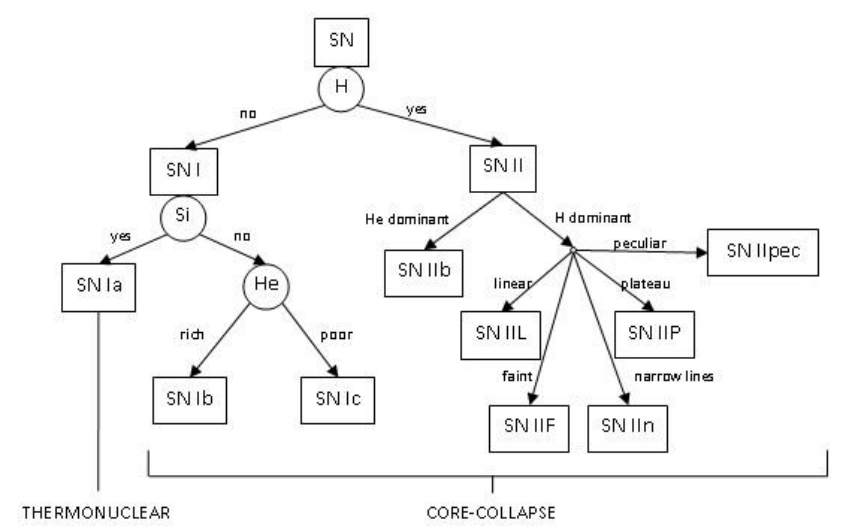

Figure 1 - Supernovae Classification Scheme [1]

The supernovae types represented by upper case are identified by light curve analysis and the supernovae types represented by lower case are identified from the spectrum analysis. However, despite the existence of classification schemes, only a few astronomer specialists are able to identify the supernova type, which makes supernovae automatic classification an alternative and, therefore, the focus of current researches.

The spectrum analysis must be done in spectra captured next the maximum light that occurs around 
two weeks after its appearance, more specifically, the analysis can be made from appearance until about one month later. The light curve analysis need to capture the luminosity daily for about 60 to 90 days before it can be analyzed, so, that analysis can only be made after this period [1]. The difference in the analysis period, led this research to focus on automatic classifiers that analyze the spectrum because the classifier developed will be installed on a telescope of the Kunlun Dark Universe Survey Telescope Project (KDUST) [3] that needs to identify the supernova type immediately after its discovery.

In the search for automatic classifiers that analyze the spectrum, two works were found and both do not consider the way in which the human expert identifies the supernova type. These works have developed the tool Supernova Identification (SNID) [4] and the tool GEneric CLAssification TOol (GELATO) [5]. These tools determine the supernova type by comparing the newly discovered spectrum with a set of spectra previously classified. The new spectrum is classified considering the type of only one spectrum that is more similar to it.

In contrast, the classifier developed in this work models the human way of analyzing the spectrum to identify the supernova type. It considers the characteristics of a spectra set for defining the supernova type and not only one spectrum. Take into consideration that a spectra set minimizes the impact in case of a change correction on the classification of a particular supernova. Changes may occur because of miss classification, as what happened in the case of a change classification correction of 26 supernovae types related by Modjaz and Blondin [6].

The basis for the development of this classifier is to model the human way of classifying supernovae. Also, to make the systematization of the classification process, to avoid the existing natural subjectivity of human classification.

Thus, this work presents a supernovae classifying automatic method that analyzes spectra captured near maximum light and using Artificial Neural Network (NN) to simulate the expert human analysis in identifying some elements that determine the supernova type.

\section{METHODS}

The automatic classifier development used two public bases of spectra available on the web to create the spectra set used for training and testing of NNs. The first found supernovae spectra base was The Online Supernova Spectrum Archive (SUSPECT), hosted at the Department of Physics and Astronomy of the University of Oklahoma, which has 1741 spectra of
185 supernovae [7]. The second found spectra base was obtained at the Harvard-Smithsonian Center for Astrophysics (CfA) site and contains 3248 spectra of 535 supernovae [8].

The spectra set was selected following these restrictions: spectra of the same supernova could only be selected from a single base; wavelength must contain the interval 3800-6800 angstroms; maximum distance from the light is -3 to +7 days. Following these restrictions 693 spectra of different supernovae types were selected, and most of them are spectrum of type la supernovae. From this set $80 \%$ of the spectra were used for training and $20 \%$ of the spectra, for testing of NNs. The training spectra set were divided into train and validation set. The spectra total set was divided as follows: 478 spectra for train, 87 spectra for validation and 128 spectra for test.

These spectra are submitted to a preprocessing based upon that done in the classifier GELATO [5] which consisted of: redshift correction; 70 angstroms smoothing each; eight angstroms each linear interpolation; normalization for a magnitude one vector.

The classifier developed identifies only the types of supernovae that are called "classic": Ia, Ib, Ic and II. The subtypes of type II were not considered for classification because there are few spectra of each of these subtypes in the used spectra base, and separating various subtypes in different classes could not produce significant results.

The modeling of the way the human expert makes visual analysis was simulated for this work. The classifier must analyzes the presence or absence of the elements hydrogen, silicon and helium in the spectrum to identify the supernovae "classic" types.

In the developing of the current classifier, which obtained the best results, the human expert information was used, highlighted in some spectra to identify the intervals used for analysis of each supernova type. Figure 2 shows an example of the highlighted intervals in spectra by human expert [6].

From this marking made by human expert in the spectra, the intervals used to identify each of the supernovae types were defined as follows:

- NN la: 5000 to 6500 angstroms;

- NN lb: 5500 to 7000 angstroms;

- NN Ic: 5500 to 6500 angstroms;

- NN II: 4.000 to 5000 and 6000 to 7.000 angstroms. 


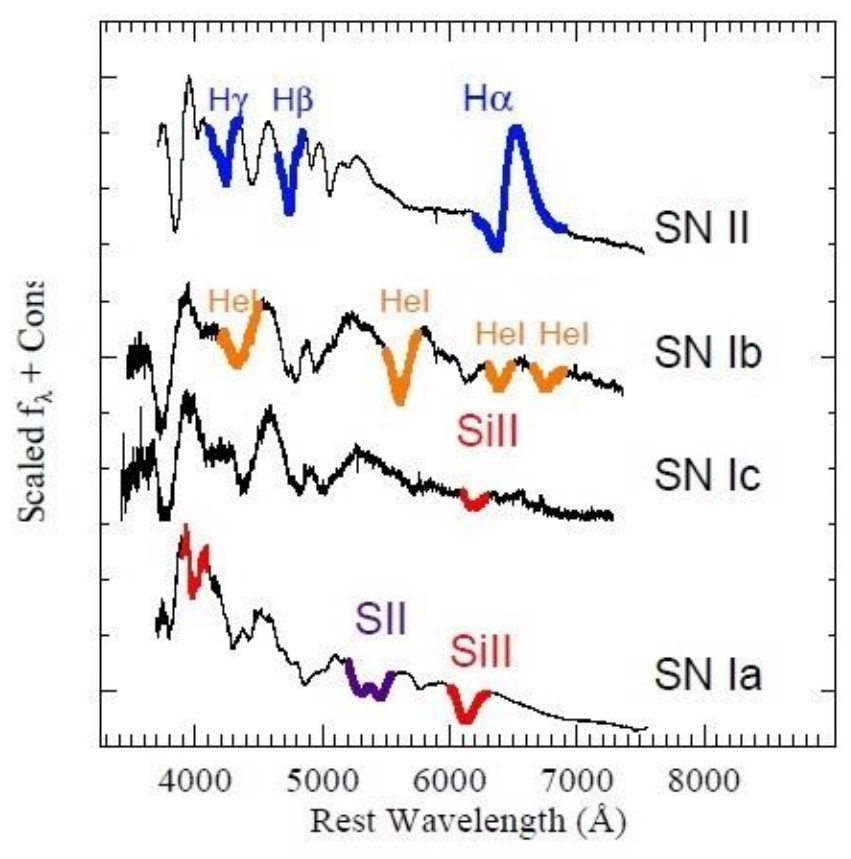

Figure 2 - Intervals examples used by human specialist [6]

The spectral classification is an appropriate task to the Multilayer Perceptron Neural Network (MLP) because it is a classification problem that can be solved using error correction learning, since it has an expected output [9]. Thus, four MLP networks were built, one $\mathrm{NN}$ for identification each one of supernova type, that is, each NN identifies only if the spectrum is of a certain type or if it is not of this type. Tests were performed varying the number of neurons and the number of layers of the NNs. The network that had the best result was a single layer with 40 neurons.

\section{RESULTS}

The test set of 128 spectra were subjected to each of the four NN. The percentage of correct answers was calculated for each NN, considering the number of spectra identified correctly in relation to the total number of spectra used in the test. The results are shown next.

\section{Type la MLP Neural Network (NN la):}

- Training epochs number $=553$

- Errors number $=1$

- Correct answers percentage $=99.2 \%$

Type Ib MLP Neural Network (NN Ib):

- Training epochs number $=1014$

- Errors number $=3$

- Correct answers percentage $=97.5 \%$

Type Ic MLP Neural Network (NN Ic):

- Training epochs number $=4769$

- Errors number $=10$

- Correct answers percentage $=91.8 \%$
Type II MLP Neural Network (NN II):

- Training epochs number $=470$

- Errors number $=2$

- Correct answers percentage $=98.4 \%$

Table 1 shows errors obtained for the 128 spectra used in testing of each of the four NNs used in the classifier. For each spectrum, that was misidentified, it shows the number that identifies the spectrum in the test (Spectrum, column 2), the name of the supernova (SN Name, column 3), the type of the supernova that has been identified by human expert (SN Type, column 4) and the supernova classification made by the neural network (NN Class, column 5). The NN class indicates whether the spectrum was classified as the type identified by the NN (Type-Xx) or has been categorized as not being that type (NotType-Xx).

Table 1 - Spectrum identification errors of the four NNs

\begin{tabular}{|c|c|c|c|c|}
\hline $\begin{array}{c}\text { Neural } \\
\text { Network }\end{array}$ & $\begin{array}{l}\text { Spec } \\
\text { trum }\end{array}$ & SN Name & $\begin{array}{l}\text { SN } \\
\text { Type }\end{array}$ & NN Class \\
\hline NN la & 121 & SN 2005cs & II & Type-la \\
\hline \multirow{3}{*}{ NN Ib } & 112 & SN 2008bo & II & Type-Ib \\
\hline & 119 & SN 1998bw & IC & Type-Ib \\
\hline & 120 & SN 1998bw & IC & Type-Ib \\
\hline \multirow{10}{*}{ NN Ic } & 55 & SN 2005A & la & Type-Ic \\
\hline & 100 & SN 2008ae & la & Type-Ic \\
\hline & 101 & SN 2008ae & la & Type-Ic \\
\hline & 114 & SN 1998bw & IC & Not-Type-Ic \\
\hline & 115 & SN 1998bw & IC & Not-Type-Ic \\
\hline & 116 & SN 1998bw & IC & Not-Type-Ic \\
\hline & 117 & SN 1998bw & Ic & Not-Type-Ic \\
\hline & 118 & SN 1998bw & IC & Not-Type-Ic \\
\hline & 119 & SN 1998bw & IC & Not-Type-Ic \\
\hline & 120 & SN 1998bw & IC & Not-Type-Ic \\
\hline \multirow{4}{*}{ NN II } & 112 & SN 2008bo & II & Not-Type-II \\
\hline & 115 & SN 1998bw & IC & Type-II \\
\hline & 119 & SN 1998bw & IC & Type-II \\
\hline & 120 & SN 1998bw & IC & Type-II \\
\hline
\end{tabular}

As can be seen in Table 1, the NN la had only one spectrum incorrectly identified. The NN lb had three errors, but two were in the same supernova. The $\mathrm{NN}$ Ic was the one that had the highest number of errors, ten errors total, but only in three different supernovas. The NN II had four errors in two different supernovas.

\section{DISCUSSION}

The tests of the classifier current version were performed on 128 spectra of 45 supernovae and, as can be seen in Table 1, only twelve spectra of five supernovas had errors in identification type: $\mathrm{SN}$ 
1998bw, SN 2005A, SN 2005cs, SN 2008ae and SN 2008bo. The supernova SN 1998bw was responsible for twelve on eighteen errors that occurred in the tests.

The most important result was the accuracy of $99.2 \%$ obtained by NN la who correctly identified all type la supernovae spectra and identified only incorrectly one type II spectrum, as type la. The errors occurred with type la supernovae were only to identify incorrectly the la as being of type Ic. This result is important because the separation of type la supernovae allows the use of the classifier as a tool to help study the universe accelerated expansion.

It is worth mentioning also that all supernovae of type $\mathrm{lb}$ were correctly identified. The error of the $\mathrm{NN} \mathrm{Ib}$ was incorrectly identified as being of type lb one type II supernovae and one of type Ic.

The use of four independent neural networks for each of the four "classical" supernovae types showed some inconsistency because four supernovae were classified as being of two different types. One possibility to solve this problem may be the creation of a fuzzy decision set of rules that indicates, in the case of inconsistency, the most likely type it belongs to.

When analyzing the computational cost of the classifier, it can be said that the computational cost required for training the neural network is very small. All neural networks were built with only two hidden layer of $20 \times 4$ neurons and did not need more than 4769 epochs to converge.

\section{CONCLUSIONS}

The supernovae automatic classifier proposed in this paper has achieved significant results that indicate the feasibility of its use for the proposed objectives. The results still need to improve, but they show that it is possible to realize the supernovae classification in places where there is no astronomer specialist or where the automatic classification is necessary.

\section{REFERENCES}

[1] C. Giunti e C. W. Kim, Fundamentals of Neutrino Physics and Astrophysics, Nova York: Oxford University Press, 2007.

[2] M. Turatto, "Classification of Supernovae," Supernovae and GammaRay Bursters, vol. 598 de Lecture Notes in Physics, pp. 21-36, 012003

[3] Chinese Center for Antarctic Astronomy, "KDUST," 2010. [Online]. Available: http://www.kdust.org/KDUST/KDUST.html. [Acesso em 25 08 2013].

[4] S. Blondin e J. L. Tonry, "Determining the Type, Redshift, and Age of a Supernova Spectrum,” The Astrophysical Journal, vol. 666, pp. 10241047, 092007.

[5] A. Harutyunyan, "Automatic Objective Classification of Supernovae,"
Padova, 2008.

[6] M. Modjaz, S. Blondin, R. P. Kirshner, T. Matheson, P. Berlind, F. B Bianco, M. L. Calkins, P. Challis, P. Garnavich, M. Hicken, S. Jha, Y. Q. Liu and G. H. Marion, "Optical Spectra of 73 Stripped-envelope Core-collapse Supernovae," The Astronomical Journal, vol. 147, pp. 99-115, 052014.

[7] M. Hogan, J. Parrent e A. Feldt, "SUSPECT," 2010. [Online]. Available: http://suspect.nhn.ou.edu/ suspect/. [Acesso em 1006 2013].

[8] Harvard-Smithsonian Center for Astrophysics (CfA), "The CfA Supernova Data Archive," 2014. [Online]. Available: https://www.cfa.harvard.edu/supernova/SNarchive.html. [Acesso em 06 06 2015].

[9] S. Haykin, Redes Neurais - Princípios e Práticas, 2a ed., Porto Alegre: Bookman, 2001 